test costs considerably less than a colonoscopy, and is cost effective.

Competing interests None.

Keywords clinical significance, cost effectiveness, faecal calprotectin, inflammatory bowel disease.

\section{PTH-026 FAECAL CALPROTECTIN: AN AUDIT TO DETERMINE ITS CLINICAL AND COST EFFECTIVENESS AT NORTH TEES AND HARTLEPOOL NHS TRUST}

doi:10.1136/gut.2011.239301.427

T Hornung, ${ }^{1, *}$ G A Anwar ${ }^{1}$ NNorthern Deanery, NHS, Newcastle upon Tyne, UK

Introduction Measurement of faecal calprotectin represents a surrogate marker of neutrophil influx into the bowel lumen, and in turn acts as a marker of intestinal inflammation. The authors aimed to determine whether faecal calprotectin testing results in changes in treatment strategy, and whether it is cost effective.

Methods A list of faecal calprotectin specimens analysed between January 2009 and April 2010 was obtained. Patient notes were reviewed by two different specialty trainees in gastroenterology to determine (1) indication for calprotectin testing, (2) result, that is, within the normal range or high, (3) outcome following the result, in terms of diagnosis made and changes in treatment strategy and (4) whether an endoscopy was avoided as a result of calprotectin testing.

A basic cost analysis was performed by calculating the total cost of the analysis of the faecal calprotectin specimens versus the total cost of the colonoscopies 'avoided' by calprotectin testing.

Results 40 patients who had a calprotectin test in the trust were included. The test was performed in two groups of patients: (1) Patients with gastrointestinal symptoms of unknown cause to help differentiate between functional and organic disease and aid in diagnosis (22 patients, or 55\%). (2) Patients known to have IBD as a marker of the disease activity (18 patients, or $44 \%$ ).

The proportion of patients with a positive faecal calprotectin in the second group (ie, IBD patients, marker of disease activity) was $61 \%$, compared to only $9 \%$ in the first group ( $p<0.001$ using $\chi^{2}$ test, DF1). Within the first group 17 subjects had a colonoscopy performed prior to the calprotectin test, which was completely normal in 13 (76\%). Of these patients none had a positive faecal calprotectin.

The use of faecal calprotectin testing in the second group of patients resulted in a change in treatment strategy in 12 out of 18 patients $(67 \%)$, compared to 0 out of 22 patients $(0 \%)$ in the first group ( $\mathrm{p}<0.001$ using $\chi^{2}$ test, DF1).

Colonoscopy was avoided in 8 patients directly as the result of faecal calprotectin testing. The tariff for day case colonoscopy at North Tees and Hartlepool NHS Foundation Trust is $£ 515$. The cost of one faecal calprotectin test is $£ 45.37$. Thus the overall cost saving for this cohort of patients is $£ 2305.20$.

Conclusion Faecal calprotectin helps to guide management and results in appropriate changes in treatment strategy when measured in patients known to have IBD as a marker of disease activity. When measured in patients with gastrointestinal symptoms to help support a diagnosis of IBS it is almost always negative, especially when the patient has had a preceding normal colonoscopy, and does not result in changes in treatment strategy. A faecal calprotectin 